\title{
Myocardial infarction in a pre-menopausal woman with angiographically normal coronary arteries
}

\author{
Ijaz A Khan, Abdul W Ansari
}

\begin{abstract}
Summary
A young pre-menopausal non-drugaddict woman without risk factors for coronary artery disease suffered from a non-Q-wave acute myocardial infarction. She presented with epigastric pain and vomiting. Diagnosis of acute myocardial infarction was not suspected at first because of her young age and lack of risk factors. She was treated for gastritis but worsening of epigastric pain and its radiation to chest warranted the diagnosis of acute myocardial infarction, which was confirmed by serial serum cardiac enzymes. Subsequent coronary angiogram revealed normal coronary arteries.
\end{abstract}

Keywords: myocardial infarction; coronary arteries

Acute myocardial infarction (AMI) with normal coronary arteries is an uncommon but well-recognised disease entity. It is primarily a disease of young adults but, due to their young age, the diagnosis of AMI may not be suspected at the time of presentation. Although myocardial infarction with normal coronary arteries has been strongly associated with smoking and use of recreational drugs with sympathomimetic actions, such as cocaine and amphetamine, its aetiology may remain unclear in many cases. We report a case of non-Q-wave AMI with normal coronary arteries in a premenopausal non-smoking non-drug-addict woman, in whom the aetiology remained unknown, and the diagnosis of AMI was not suspected initially due to her young age and lack of risk factors.

\section{Case report}

A 43-year-old woman was brought to the emergency room with complaints of epigastric pain of one-hour duration. Pain was severe in intensity, constricting in nature and was radiating upwards to the lower part of sternum. It was associated with nausea, vomiting, diaphoresis and dizziness. She did not have a history of hypertension, diabetes mellitus or any other disease. She had never smoked cigarettes or used recreational drugs. She was neither pregnant nor using oral contraceptives. There was no family history significant for coronary artery disease. On physical examination, she was in pain and was anxious. Besides tachycar- dia, her physical examination, including abdominal examination, was unremarkable. Electrocardiogram (ECG) demonstrated sinus tachycardia, but was otherwise normal. Because of the epigastric pain and vomiting, she was diagnosed as a case of gastritis and was treated with antacids and intravenous fluids. Pain continued to worsen with more radiation to the chest. The patient became more diaphoretic, apprehensive and tachycardic. Because of increasing chest pain and diaphoresis, a diagnosis of AMI was suspected. Oral aspirin and intravenous nitroglycerine and heparin were administered and the patient was transferred to the coronary care unit. The ECG, which was subsequently repeated many times, did not reveal any evidence of myocardial ischaemia or infarction. The patient started improving while on nitroglycerine infusion and soon became pain-free.

Serum cardiac enzymes were measured on suspicion of AMI and subsequently eight hourly. The first set of cardiac enzymes revealed markedly elevated serum levels; creatine kinase (CK) was $1103 \mathrm{IU} / 1$ (normal range 25-215), $M B$ isoenzyme of creatine kinase (CK-MB) was $110 \mathrm{ng} / \mathrm{ml}(0-4)$, MB fraction was $10 \%(0-4 \%)$ and aspartate transaminase was $83 \mathrm{IU} / 1(0-40)$. The second set demonstrated even higher values; CK $1489 \mathrm{IU} / 1$, CK-MB $160 \mathrm{ng} / \mathrm{ml}, \mathrm{MB}$ fraction $11 \%$ and aspartate transaminase $70 \mathrm{IU} / 1$. Thereafter, serum levels of cardiac enzymes started falling and third, fourth and fifth sets revealed $\mathrm{CK}$ 1073, 533 and 400 IU/1, CK-MB 100, 34 and $21 \mathrm{ng} / \mathrm{ml}, \mathrm{MB}$ fraction 9, 6 and 5\% and aspartate transaminase 66,59 and $41 \mathrm{IU} / 1$, respectively. Serum cardiac enzymes drawn on the third day of admission demonstrated values within normal limits, with CK 154 IU/1, CK-MB $3 \mathrm{ng} / \mathrm{ml}$, MB fraction $2 \%$ and aspartate transaminase $22 \mathrm{IU} / 1$. This typical rise and fall in serum cardiac enzymes, along with the clinical presentation, was diagnostic of AMI. All other laboratory tests including serum cholesterol and triglycerides, liver function tests, kidney function tests and blood counts were well within normal limits. Twodimensional echocardiogram revealed no wall motion abnormalities with normal left ventricular ejection fraction. The patient went through an uneventful hospital course free of complications. Because her age and lack of risk factors, cardiac catheterization was performed one week after the AMI. Coronary angiography 
demonstrated normal coronary arteries and left ventriculogram demonstrated preserved wall motion and systolic function. Provocation tests for spasm were not performed during coronary angiography. She was discharged on aspirin, isosorbide dinitrate and amlodipine, a calcium channel blocker.

\section{Discussion}

AMI with normal coronary arteries may be due to various causes but the exact aetiology often remains unknown. It has been reported in a variety of non-atherosclerotic disorders including thromboembolism, atrial myxoma, aortic stenosis, trauma, congenital coronary anomalies, carbon monoxide poisoning, severe sepsis and hypercoagulable states, like polycythemia, sickle cell anaemia, thrombocytosis and thrombotic thrombocytopenic purpura. In premenopausal women, it has been reported in association with high progestational states, such as pregnancy, puerperium and oral contraceptive use. However, the strongest association has been found with smoking ${ }^{1}$ and use of recreational drugs with sympathomimetic actions, such as cocaine ${ }^{2}$ and amphetamines. ${ }^{3}$ The suggested underlying mechanism in these cases, and in cases of unknown aetiology, is coronary vasospasm with superimposed thrombosis, as this has been clearly demonstrated in many animal and human angiographic studies. ${ }^{4}$ Clinically, AMI with normal coronary arteries has been associated with other vasospastic syndromes, such as migraine and Raynaud's phenomenon. Coronary spasm may cause prolong blood stasis and injury to underlying vascular endothelium, which may lead to activation of coagulation factors and platelets, causing thrombosis, and resulting in coronary occlusion and AMI in the absence of anatomically occlusive lesion in the coronary artery. The trigger events which precipitate vasospasm and thrombosis in predisposed individuals are not always clear, but intense autonomic nervous system activation, for example, during marked exertion or intense emotional turmoil, has been reported to cause AMI with normal coronary arteries.

Most patients with AMI and normal coronary arteries are young, ${ }^{6}$ with a mean age around 40 years, and do not usually have any medical history of angina pectoris or myocardial infarction. They present in the similar way to patients with coronary atherosclerosis but the diagnosis can be easily overlooked due to their younger

1 McKenna WJ, Chew CY, Oakley CM. Myocardial infarction with normal coronary angiogram. Possible mechanism of smoking risk in coronary artery disease. $\mathrm{Br}$ Heart $\mathcal{J}$ $1980 ; 43: 493-8$

2 Amin M, Gabelman G, Karpel J, Buttrick P. Acute myocardial infarction and chest pain syndrome after cocaine use. Am $\mathcal{f}$ Cardiol 1990;66:1434-7.

3 Carson P, Oldroyd K, Phadke K. Myocardial infarction due to amphetamine. BMF 1987;294:1525.

4 Vincent GM, Anderson JL, Marshall HW. Coronary spasm producing coronary thrombosis and myocardial infarction. N Engl F Med 1983;309:220-3.

5 Chierchia S. The role of alpha-adrenergic receptors in the pathogenesis of coronary spasm. Clin Cardiol 1983;6:496500 .

\section{Learning point}

In pre-menopausal women with no risk factors for coronary artery disease and normal coronary arteries, myocardial infarction can still occur age, absence of recognisable coronary risk factors, and lack of history of ischaemic heart disease. The most common ECG feature is ST segment elevation but the ECG can be normal or near normal if the myocardial infarction is of non-Q-wave type. Infarction tends to be smaller and the occurrence of complications, such as hypotension, heart failure, cardiogenic shock and malignant arrhythmia is generally less. $^{7}$

The treatment of AMI with normal coronary arteries is similar to that with coronary atherosclerosis and thrombolytic therapy should be given if not contraindicated. The diagnosis of normal coronary anatomy manifests on subsequent coronary angiogram, although it may be suspected in certain circumstances, for example, in a young cocaine addict with no risk factors for coronary artery disease. Once normal coronary arteries are identified on angiogram, a calcium channel blocker with coronary vasodilator properties should be added to the treatment regimen because coronary vasospasm plays a primary role in the pathophysiology of AMI with normal coronary arteries. ${ }^{8}$ The prognosis of AMI with normal coronary arteries is generally good and recurrent infarction, post-infarction angina, heart failure and sudden cardiac death are unusual..$^{9}$ Because of the lack of flow-limiting lesions in the coronary arteries, stress ECG and imaging methods are not usually helpful prognostic tools. Long-term survival mainly depends on residual left ventricular function, which is usually preserved.

This non-smoker non-drug-addict young female without risk factors for coronary artery disease suffered from a non-Q-wave AMI of obscure aetiology and subsequently was revealed to have angiographically normal coronary arteries. She did not have any complications and her residual left ventricular function was preserved. The diagnosis of AMI was not suspected initially because of her young age and lack of risk factors for coronary artery disease.

6 Betriu A, Pare JC, Sanz GA, et al. Myocardial infarction with normal coronary arteries. Am $\mathcal{F}$ Cardiol 1981;48:28-32.

7 Pathe M, Baron B, Gharib A, et al. Myocardial infarction with normal coronary angiography. Apropos of 9 cases. A retrospective and comparative study. Ann Cardiol Angiol 1996;45:12-7.

8 Bott-Silverman C, Heupler FA. Natural history of pure coronary spasm in patients treated medically. $₹ \mathrm{Am}$ Coll Cardiol 1983;2:200-5.

9 Raymond R, Lynch J, Underwood D, Leatherman J, Razavi $M$. Myocardial infarction and normal coronary arteriography: a 10 year clinical and risk analysis of 74 patients. F Am Coll Cardiol 1988;11:471-7. 